\title{
Geometric and electronic structures of corannulene polymers: Ultra narrow graphene ribbons with corrugation and topological defects
}

\author{
Kohei Narita*, Susumu Okada** \\ Graduate School of Pure and Applied Sciences, University of Tsukuba, 1-1-1 Tennodai, \\ Tsukuba, Ibaraki 305-8571, Japan
}

\begin{abstract}
We used density functional theory to study the geometric and electronic structure of dimerized and one-dimensionally polymerized corannulene as ultra-narrow graphene ribbons with corrugation and topological defects. Our computations reveal that the relative stability and electronic structure of dimerized and polymerized corannulene are sensitive to the intermolecular covalent networks. The energy gap between the highest occupied and lowest unoccupied states of corannulene dimers is narrower than that of isolated corannulene. The corannulene polymers are semiconductors with a direct energy gap of about $1 \mathrm{eV}$ depending on intermolecular bonds. The polymers possess moderate mechanical stiffness having Young's moduli of $200 \mathrm{GPa}$.
\end{abstract}

Keywords: Corannulene, Graphene nanoribbon, One dimensional corannulene polymer, Electronic structure

\section{Introduction}

Over the past decade, graphene has attracted much attention in nanoscale science and technology research, because of the unique physical and chemical properties that arise from the honeycomb network of $\mathrm{sp}^{2} \mathrm{C}$ atoms with atom thickness $[1,2,3,4,5,6,7,8]$. Because of its metallic electronic

\footnotetext{
*TEL/FAX: +81-298535600 (ext. 8233)/+81-298535924

**TEL/FAX: +81-298535921/+81-298535924

Email addresses: knarita@comas.frsc.tsukuba.ac.jp (Kohei Narita), sokada@comas.frsc.tsukuba.ac.jp (Susumu Okada)
} 
structure and vanishing density of state at the Fermi level [9, 10], graphene derivatives exhibit variations in their electronic structure depending on the boundary conditions imposed upon the two-dimensional honeycomb network. For instance, an open boundary condition on graphene results in graphene ribbons with nanometer width (graphene nanoribbons or GNR). The electronic structure of GNRs strongly depends on their width and the atomic arrangement at the ribbon edge. GNRs with armchair edges are semiconductors in which the band gap oscillates with respect to the ribbon width and asymptotically decreases as the ribbon width increases because of the discretization conditions imposed on the two-dimensional hexagonal Brillouin zone of graphene $[11,12,13]$. In contrast, GNRs with zigzag edges are metals with peculiar edge-localized states known as edge states, which lead to spin polarization at the edge atomic sites $[11,12,14,15,16]$. In addition, it has been pointed out that the edge roughness also causes further variation in electronic properties of GNRs [17, 18].

In accordance with the variations in the electronic structure of GNRs, GNRs are applicable as constituent materials for future electronic and spintronic devices. On the other hand, because their electronic structure depends sensitively on the geometric structure, precise structural control is mandatory for practical applications. For such purposes, cyclodehydrogenation of polycyclic aromatic hydrocarbon (PAH) molecules is one of the plausible procedures for synthesizing structurally well-controlled GNRs [19, 20]. The most important advantage of this synthetic technique is that the sizes and shapes of the resultant GNRs are, in principle, atomically controllable by assembling appropriate PAH molecules under optimum external conditions. For instance, oligomerization and polymerization of coronene molecules $\left(\mathrm{C}_{24} \mathrm{H}_{12}\right)$ have been experimentally demonstrated inside carbon nanotubes(CNTs), and can be regarded as a prototype of ultra narrow GNRs with edge roughness $[21,22]$. In addition to coronene [23, 24], corannulene [25, 26] has been encapsulated into CNTs [27]. Encapsulated corannulene may form one-dimensional polymeric structures via cyclodehydrogenation in a similar manner to coronene in CNTs, and can be regarded as narrow GNRs with structural corrugation and topological defects.

In this work, we investigate the geometric and electronic structures of polymerized corannulene as a representative prototype of GNRs having structural corrugation and topological defects, because the curvature and the topological defects in $\mathrm{sp}^{2}$ hexagonal networks occasionally induce the modulation of electronic structure near the Fermi level $[28,29]$. Our density functional 
theory study reveals that the electronic structure of the polymerized corannulene sensitively depends on the intermolecular network topology but is independent of the corrugation. Furthermore, the polymers exhibit moderate mechanical strength, having a Young's modulus of about 200GPa although the polymers possess pentagonal rings. In addition, we explore the properties of corannulene dimers as polymer precursors and find that dimerization leads to a substantial decrease in the energy gap between the highest occupied and lowest unoccupied states.

\section{Calculation methods}

All calculations were performed using the density functional theory (DFT) [30, 31] as implemented within the Simulation Tool for Atom TEchnology (STATE) package [32]. To calculate the exchange-correlation energy among interacting electrons, we used the generalized gradient approximation (GGA) including the spin degree of freedom with the functional forms of PerdewBurke-Ernzerhof (PBE) [33]. Ultrasoft pseudopotentials generated using the Vanderbilt scheme were employed to describe the interaction between the valence electrons and ions. The valence wave functions and charge density were expanded in terms of the plane-wave basis set with cutoff energies of 25 and 225 Ry, respectively. To simulate an isolated corannulene dimer, we used a super cell scheme with a cell size of $31 \times 16 \times 16 \AA$. For the isolated corannulene polymer, the polymer is separated from its periodic images by at least $10 \AA$ vacuum regions. Integration over the Brillouin zone was carried out using the $\Gamma$ point and an equidistance mesh of $4-k$ points for corannulene dimers and polymers, respectively. Structural optimization was performed until the remaining force acting on each atom was less than $5 \mathrm{mRy} / \AA$ for each lattice constant.

\section{Results and discussion}

In our exploration of possible polymeric structures, we start with an investigation of corannulene dimer structures because of the variety of possible molecular arrangements subsequent to cyclodehydrogenation. Figure 1 shows optimized structures of the corannulene dimer considered here, in which the corannulene molecules are connected via hexagonal, pentagonal, and tetragonal intermolecular rings. In addition, because of the bowl shape of corannulene, we further consider three dimer conformations, the wavy, flat, 


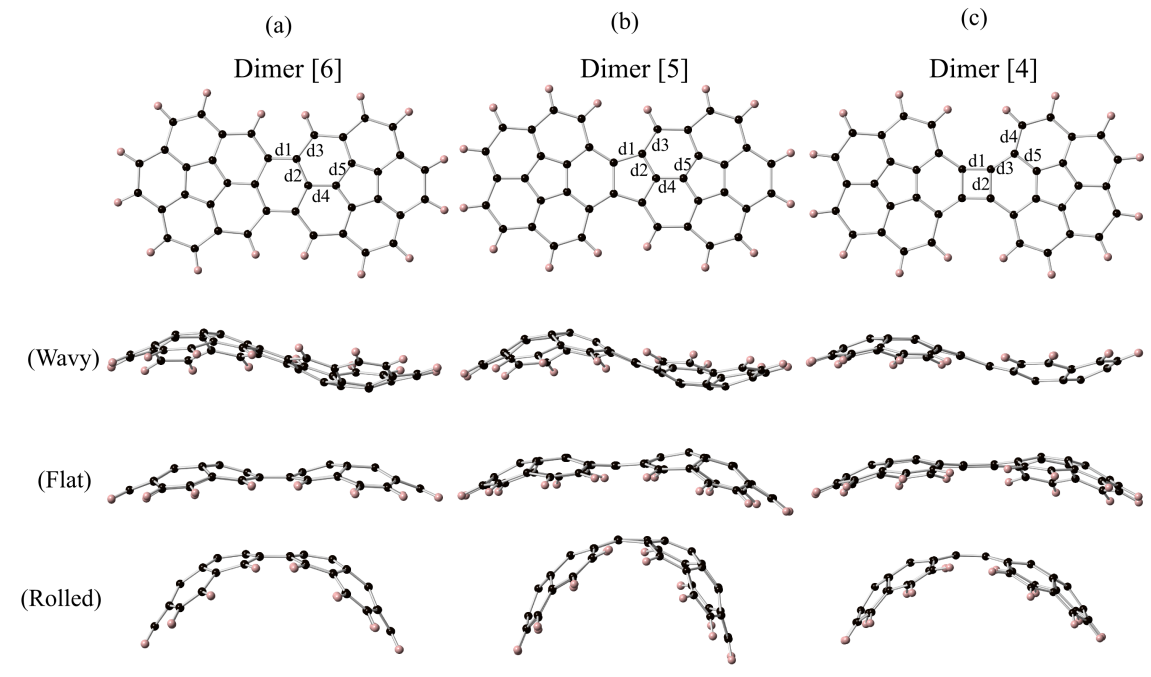

Figure 1: Top and side views of geometric structures of the corannulene dimer in which corannulene molecules are connected via (a) six-membered, (b) five-membered, and (c) four-membered rings.

and rolled conformations for each dimer (Fig. 1). Corannulene molecules are alternately arranged in the wavy conformation. Corannulene bowls are aligned in the same orientation without and with curvature for the flat and rolled conformations, respectively.

The optimized bond lengths of corannulene dimer bond lengths are 1.35 - $1.41 \AA$ within the corannulene unit which is nearly identical to those in an isolated corannulene molecule $(1.37-1.40 \AA$ ) (Table. 1). On the other hand, for the intermolecular bonds, the optimized lengths are about 1.46, 1.47, and $1.50 \AA$ for hexagonal, pentagonal, and tetragonal rings, respectively, which are longer than the bond lengths in typical $\mathrm{sp}^{2}$ materials. The bond length associated with the intermolecular rings strongly depends on the intermolecular bond morphology. The bond length increases as bond morphology is distorted further from an ideal $\mathrm{sp}^{2}$ bond.

The relative energies of three various corannulene dimers are summarized in Table 2. Energies are measured relative to that of the ground state structure of the dimer possessing a hexagonal intermolecular ring and the wavy 
Table 1: Bond length of corannulene dimers. Bond indexes are listed in Fig. 1.

\begin{tabular}{cccccc}
\hline$(\AA)$ & $\mathrm{d} 1$ & $\mathrm{~d} 2$ & $\mathrm{~d} 3$ & $\mathrm{~d} 4$ & $\mathrm{~d} 5$ \\
\hline $6 \mathrm{~W}$ & 1.45 & 1.44 & 1.39 & 1.36 & 1.40 \\
$6 \mathrm{~F}$ & 1.45 & 1.44 & 1.39 & 1.36 & 1.40 \\
$6 \mathrm{R}$ & 1.46 & 1.44 & 1.39 & 1.38 & 1.41 \\
\hline $5 \mathrm{~W}$ & 1.46 & 1.42 & 1.39 & 1.35 & 1.4 \\
$5 \mathrm{~F}$ & 1.47 & 1.41 & 1.39 & 1.34 & 1.4 \\
$5 \mathrm{R}$ & 1.47 & 1.42 & 1.38 & 1.36 & 1.41 \\
\hline $4 \mathrm{~W}$ & 1.49 & 1.42 & 1.4 & 1.44 & 1.39 \\
$4 \mathrm{~F}$ & 1.51 & 1.41 & 1.41 & 1.44 & 1.39 \\
$4 \mathrm{R}$ & 1.52 & 1.41 & 1.41 & 1.44 & 1.4 \\
\hline
\end{tabular}

conformation. The corannulene dimer with a hexagonal intermolecular ring has lower total energy by about 0.4 and $1.1 \mathrm{eV}$ than those with a pentagonal and tetragonal intermolecular ring, respectively. In contrast, the energy of the dimer is less sensitive to the molecular conformations than the intermolecular ring: For each dimer with the intermolecular ring, the wavy conformation has the lowest total energy among the three molecular conformations studied here. The total energy of the wavy conformation is lower by about $0.1-0.2 \mathrm{eV}$ than those of the flat and rolled conformations. This indicates that the energetics of the corannulene dimers primary depend on the nature of the intermolecular covalent rings.

Because the wavy conformation is the ground state molecular conformation for all dimers, we investigate the electronic structure of dimers possessing hexagonal, pentagonal, and tetragonal rings with the wavy conformations. Figure 2 shows the electronic structure of corannulene dimers with the wavy conformation. The calculated energy gap between the highest occupied (HO) and the lowest unoccupied (LU) Kohn-Sham states is 2.03, 1.80, and 1.12 $\mathrm{eV}$ for the dimers with the hexagonal, pentagonal, and tetragonal rings, respectively. The HO-LU gap, calculated using DFT with the local density approximation, of an isolated corannulene is $3.01 \mathrm{eV}$, indicating that the formation of intermolecular bonds causes a decrease in the HO-LU gap. The dimers with the other molecular conformations possess almost the same HOLU gap as the dimer with the wavy conformation (Table 2).

Figure 3 shows the squared wave functions of the $\mathrm{HO}$ and LU states of three corannulene dimers. The HO and LU states of all corannulene dimers 

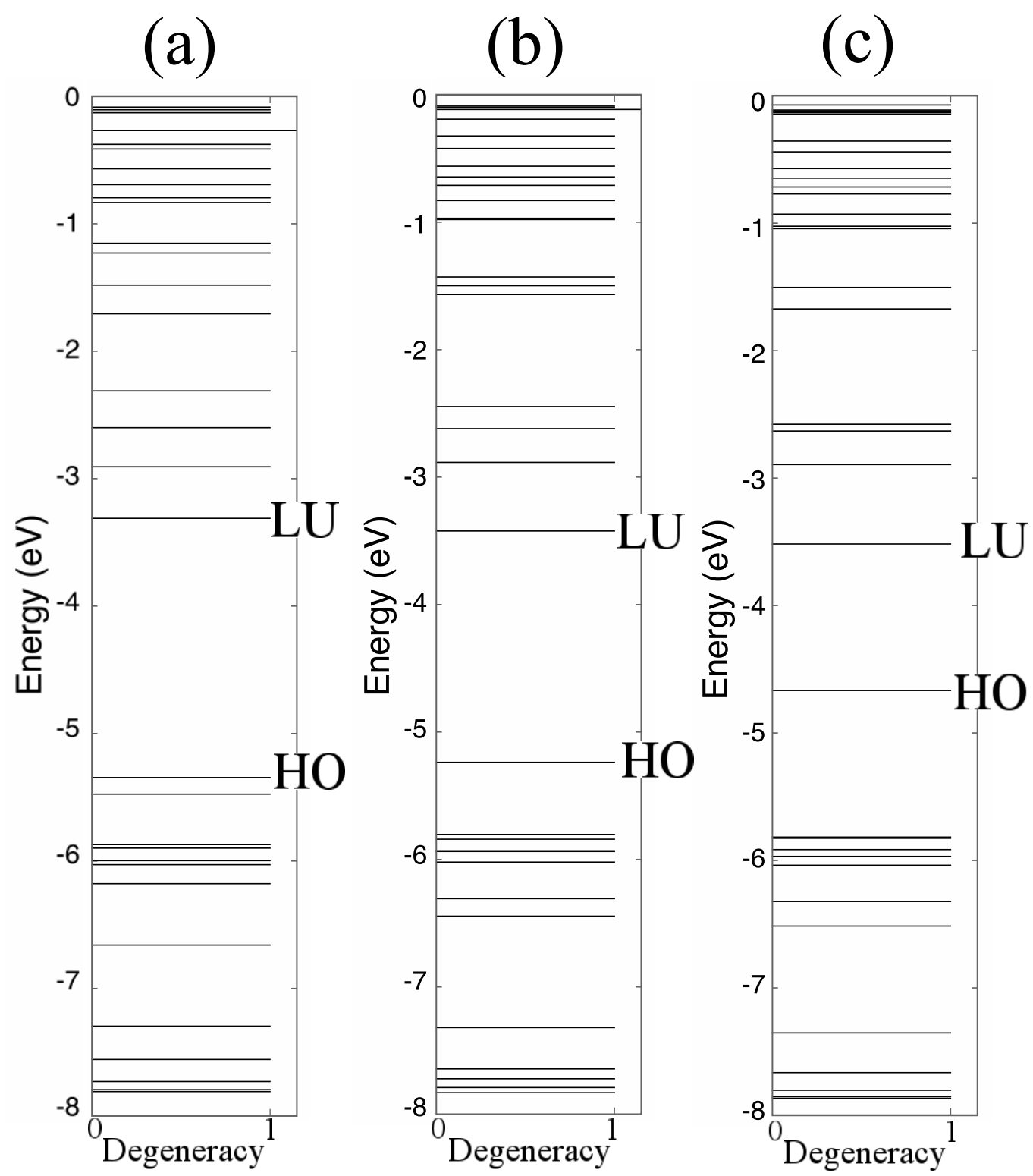

Figure 2: Electronic structures of the corannulene dimers in which corannulene molecules are connected via (a) hexagonal, (b) pentagonal, and (c) tetragonal rings. Energies are measured relative to the vacuum level. 
Table 2: Relative total energies and HO-LU gaps of corannulene dimers. Energies are measured relative to that of the ground state dimer containing a hexagonal ring between the corannulene molecules.

\begin{tabular}{ccc}
\hline & Energy $(\mathrm{eV} /$ corannulene $)$ & HO-LU gap $(\mathrm{eV})$ \\
\hline $6 \mathrm{~W}$ & 0 & 2.03 \\
$6 \mathrm{~F}$ & 0.18 & 1.93 \\
$6 \mathrm{R}$ & 0.14 & 1.88 \\
\hline $5 \mathrm{~W}$ & 0.37 & 1.80 \\
$5 \mathrm{~F}$ & 0.5 & 1.80 \\
$5 \mathrm{R}$ & 0.63 & 1.80 \\
\hline $4 \mathrm{~W}$ & 1.14 & 1.12 \\
$4 \mathrm{~F}$ & 1.23 & 1.12 \\
$4 \mathrm{R}$ & 1.15 & 1.12 \\
\hline
\end{tabular}

exhibit antibonding and bonding nature with respect to the intermolecular covalent bonds. For all dimers, the LU state possesses a nodal line along the dimer axis. On the other hand, while the HO state of the dimers with the hexagonal ring possesses a nodal line with respect to the dimer axis, the $\mathrm{HO}$ state of the dimers with pentagonal and tetragonal ring display different distributions. The wave function has nodal lines normal to the dimer axis. The distribution of the $\mathrm{HO}$ state of the dimers with the pentagonal and tetragonal rings is different from that of the isolated corannulene. Thus, dimerization modulates the wave function distribution of the $\mathrm{HO}$ state of the isolated corannulene molecule.

Because the wavy conformation is most energetically favorable toward intermolecular bond formation between corannulene by cyclodehydrogenation, we focus on one-dimensionally polymerized corannulene chains with the wavy conformation in which the polymer contains pentagonal rings as the intermolecular bonds (Fig. 4(a): polymer[55]) and the polymer contains tetragonal and hexagonal rings alternately as the intermolecular bonds ( Fig. 4(b): polymer[46] ). The optimum intermolecular distances are 7.22 and 7.24 $\AA$ for the polymer[55] and polymer[46], respectively. Under the optimum intermolecular distance, the optimized bond lengths of corannulene polymers are $1.35-1.44 \AA$ within the corannulene unit which is almost the identical to those in the corannulene and its dimers. For the intermolecular bonds, optimized values are $1.45,1.42$, and $1.50 \AA$ for pentagonal, hexagonal, and 
(a)

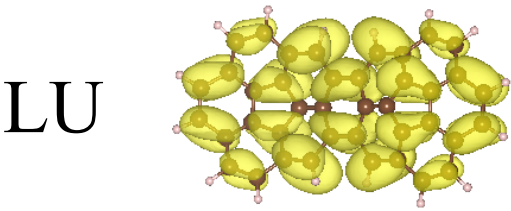

$\mathrm{HO}$ (b)

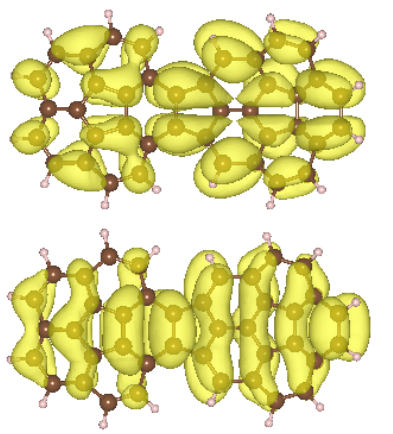

(c)

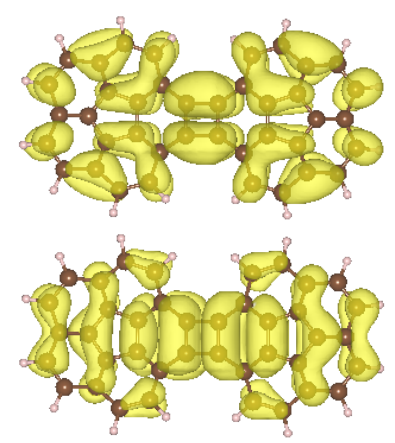

Figure 3: (Color online) Squared wave functions of the HO and LU states of corannulene dimer isomers with (a) hexagonal, (b) pentagonal, and (c) tetragonal intermolecular rings.

tetragonal rings, respectively (Table 3 ). The bond length is identical to that in the dimer structure except for the bond length of the hexagonal ring. In this case, the bond is slightly shorter than that in the dimer.

Table 3: Bond lengths in corannulene polymers. Bond indexes are listed in Fig. 4.

\begin{tabular}{ccccccccccc}
\hline$(\AA)$ & $\mathrm{d} 1$ & $\mathrm{~d} 2$ & $\mathrm{~d} 3$ & $\mathrm{~d} 4$ & $\mathrm{~d} 5$ & $\mathrm{~d} 6$ & $\mathrm{~d} 7$ & $\mathrm{~d} 8$ & $\mathrm{~d} 9$ & $\mathrm{~d} 10$ \\
\hline polymer[55] & 1.45 & 1.42 & 1.38 & 1.41 & 1.41 & - & - & - & - & - \\
polymer[46] & 1.50 & 1.40 & 1.38 & 1.44 & 1.41 & 1.42 & 1.44 & 1.39 & 1.35 & 1.39 \\
\hline
\end{tabular}

Table 4: Relative total energies of corannulene polymers. Energies are measured relative to the ground state polymer containing pentagonal rings between corannulene molecules.

\begin{tabular}{lcc}
\hline & Energy $(\mathrm{eV} /$ corannulene) & Young's modulus $(\mathrm{GPa})$ \\
\hline polymer[55] & 0 & 200 \\
polymer[46] & 0.38 & 242 \\
\hline
\end{tabular}

Table 4 shows the relative energies and Young's modulus of corannulene polymers. The total energy of polymer[46] is about $0.4 \mathrm{eV}$ higher than that of polymer[55]. The higher total energy of polymer[46] is ascribed to the tetragonal intermolecular ring whose energy is higher than the energies of the hexagonal and pentagonal rings by 1.1 and $0.7 \mathrm{eV}$, respectively. Furthermore, 

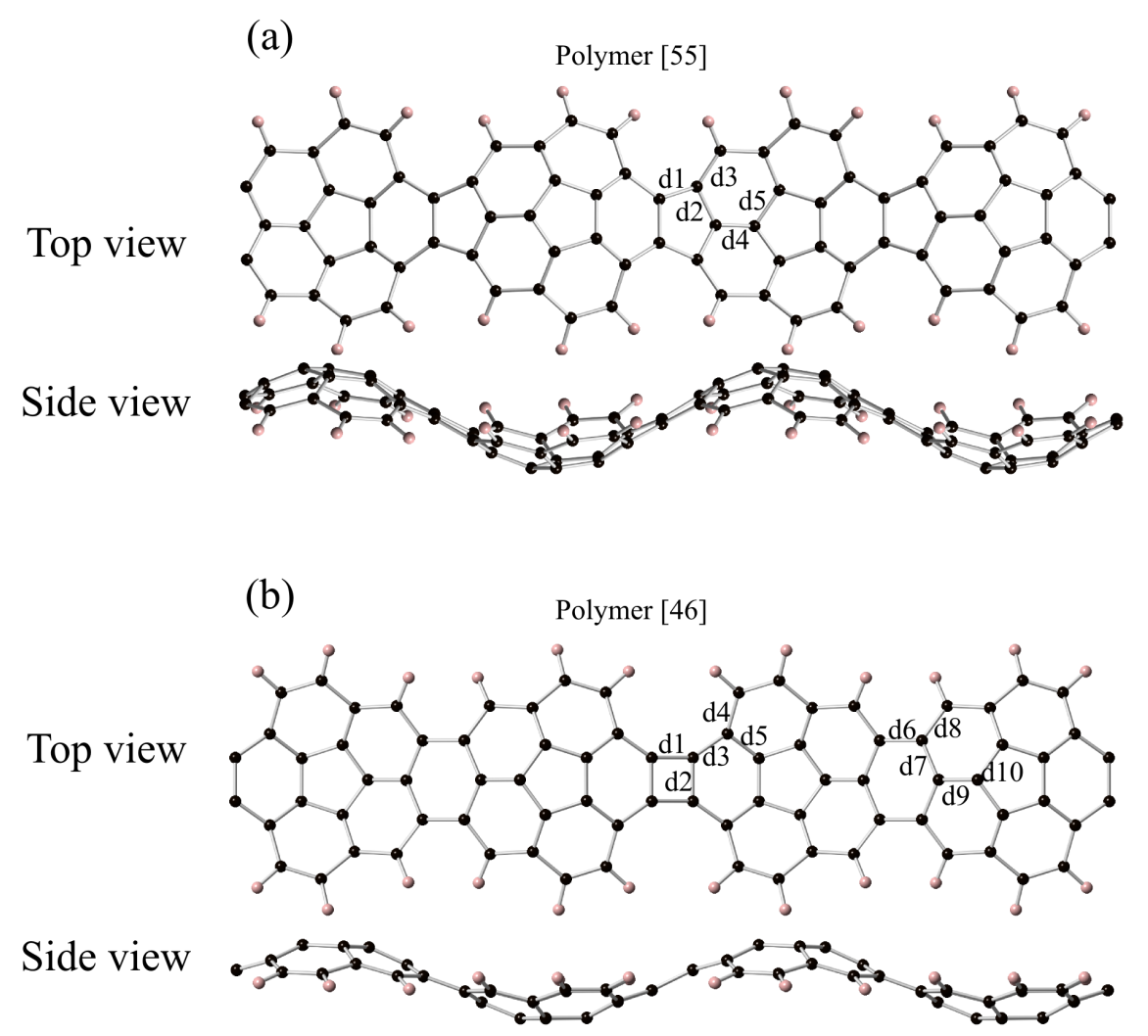

Figure 4: Top and side views of geometric structures of (a) polymer[55] and (b) poly$\operatorname{mer}[46]$. 
interestingly, this energy difference corresponds to the simple sum of the relative stability of the intermolecular bonds evaluated in the corannulene dimer. Thus, the higher total energy of polymer[46] is caused by the large strain arising from the tetragonal ring. We also calculated Young's modulus of the corannulene polymers. As shown in Table 4, the Young's modulus is 200 and $242 \mathrm{GPa}$ for polymer[55] and polymer[46], respectively, indicating that the corannulene polymers possess moderate mechanical stiffness despite the polymer containing structural corrugation and topological defects. On the other hand, because of these topological defects, the calculated values for the polymers are smaller than that of GNR with similar width [34].

Figure 5 shows the electronic energy band of corannulene polymers. Both polymers are semiconductors with the direct energy gap at $\Gamma$ point of 0.97 and $0.51 \mathrm{eV}$ for polymer[55] and polymer[46], respectively. The gap is considerably narrower than armchair GNRs with the same ribbon width. We also find that the band width near the fundamental gap is narrower than that of the pristine ribbons. In particular, the $\mathrm{HO}$ and the second highest occupied states of polymer[46] possess narrow band widths of 0.5 and 0.1 $\mathrm{eV}$, respectively, indicating the possibility that polymer[46] exhibits unusual physical phenomena by injecting holes into these bands. The polymer[46] with appropriate hole density may cause spin polarization or charge density wave on the polymer network. In addition to the electronic structure near the gap, the electronic structure of the polymers is totally different from that of graphene nanoribbons with armchair or zigzag edges because of topological defects in the polymeric networks. The electronic energy band of the polymers is qualitatively insensitive to the structural corrugation, as evidenced by results of calculations on the polymers under tensile strains that have nearly planar network structures [Figs. 5(c) and 5(d)].

Figure 6 shows the squared wave functions of the HO and LU states of the corannulene polymers. The LU state possesses nodal lines parallel to the polymer axis, as in the case of their dimer structures. In contrast, for the HO states, the wave function possesses nodal lines normal to the polymer axis. Furthermore, for the HO state of polymer[46], the wave function distribution on the hexagonal rings is smaller than that on the tetragonal rings, indicating weak segmentation of the $\pi$ electrons. 
(a)

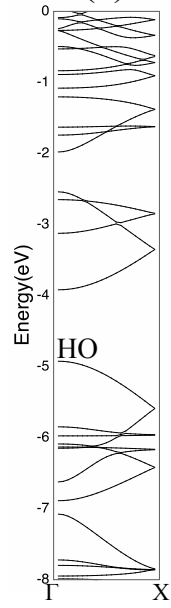

(c)

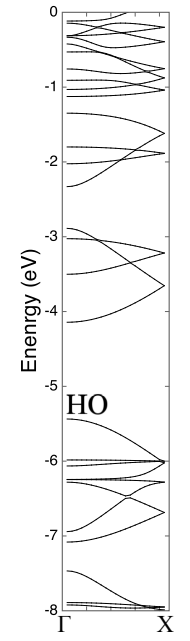

(b)

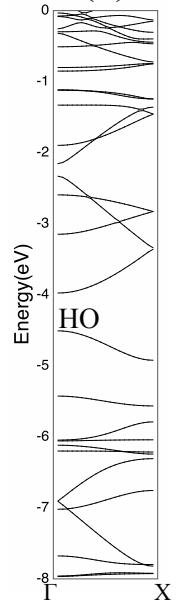

(d)

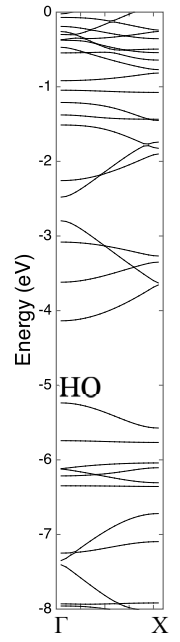

Figure 5: Electronic energy bands of (a) polymer[55] and (b) polymer[46] rings under the equilibrium lattice constatnt. Electronic energy bands of (c) polymer[55] and (d) polymer[46] under the $10 \%$ tensile strain. HO denotes the highest branch of the valence band. Energies are measured relative to the vacuum level. 
(a)

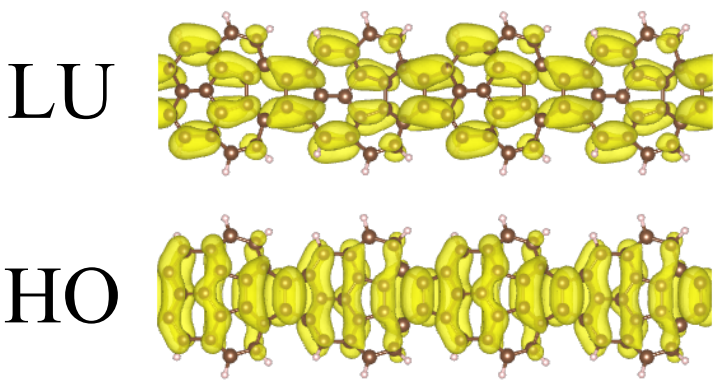

(b)
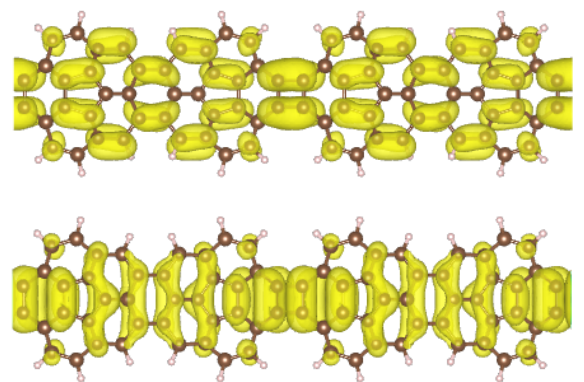

Figure 6: Squared wave functions of the HO and LU states of corannulene polymers: (a) polymer[55] and (b) polymer[46].

\section{Conclusion}

We studied the geometric and electronic structures of dimerized and onedimensionally polymerized corannulene using first-principles total-energy calculations within the density functional theory framework. Our calculations reveal that the corannulene dimer has nine possible geometric structures. Among the corannulene dimer conformations studied here, the dimer with a wavy conformation and hexagonal ring is the most stable. Dimerization causes a decrease in the energy gap between the highest occupied and lowest unoccupied states of the corannulene dimer relative to that of an isolated corannulene molecule. In the case of corannulene polymers, the corannulene polymer with pentagonal rings is more stable than the polymer with hexagonal and tetragonal rings, because the tetragonal ring considerably increases the total energy due to the highly distorted intermolecular bonds. All polymers are semiconductors with the direct energy gap at $\Gamma$ point of 0.97 and $0.51 \mathrm{eV}$ for polymer [55] and polymer[46], respectively. Both polymers possess moderate stiffness of which Young's modulus are about $200 \mathrm{GPa}$.

\section{Acknowledgements}

This work was supported in part by a Grant-in-Aid for Scientific Research from the Ministry of Education, Culture, Sports, Science and Technology of Japan and the Joint Research Program on Zero-Emission Energy Research, Institute of Advanced Energy, Kyoto University. Computations were performed on a NEC SX-8/4B at the University of Tsukuba, a SGI ICE XA/UV 
at the Institute for Solid State Physics, The University of Tokyo, and a NEC SX-Ace at the Cybermedia Center, Osaka University.

\section{References}

[1] K. S. Novoselov, A. K. Geim, S. V. Morozov, D. Jiang, M. I. Katsnelson, I. V. Grigorieva, S. V. Dubonos, and A. A. Firsov, Nature 438 (2005) 197.

[2] Y. Zhang, Y. -W. Tan, H. L. Stormer, and P. Kim, Nature 438 (2005) 201.

[3] X. Du, I. Skachko, F. Duerr, A. Luican, and E. Y. Andrei, Nature 462 (2009) 192.

[4] K. I. Bolotin, F. Ghahari, M. D. Shulman, H. L. Stormer, and P. Kim, Nature 462 (2009) 196.

[5] K. S. Novoselov, Z. Jiang, Y. Zhang, S. V. Morozov, H. L. Stormer, U. Zeitler, J. C. Maan, G. S. Boebinger, P. Kim, and A. K. Geim, Science 315 (2007) 1379.

[6] K. S. Novoselov, E. McCann, S. V. Morozov, V. I. Fal'ko, M. I. Katsnelson, U. Zeitler, D. Jiang, F. Schedin, and A. K. Geim, Nature, Phys. 2 (2006) 177.

[7] S. V. Morozov, K. S. Novoselov, and A. K. Geim, Phys. Rev. Lett. 97 (2006) 016801.

[8] M. I. Katsnelson, K. S. Novoselov, and A. K. Geim, Nature, Phys. 2 (2006) 620.

[9] M. S. Dresselhaus and G. Dresselhaus, Adv. Phys. 30 (1981) 139.

[10] M. S. Dresselhaus and G. Dresselhaus, Adv. Phys. 51 (2002) 1.

[11] M. Fujita, K. Wakabayashi, K. Nakada, and K. Kusakabe, J. Phys. Soc. Jpn. 65 (1996)1920.

[12] K. Nakada, M. Fujita, G. Dresselhaus, and M. S. Dresselhaus, Phys. Rev. B 54 (1996) 17954. 
[13] Y. -W. Son, M. L. Cohen and S. G. Louie, Phys. Rev. Lett. 97 (2006) 216803.

[14] Y. Miyamoto, K. Nakada, M. Fujita, Phys. Rev. B 59 (15) (1999) 98589861.

[15] Y. Kobayashi, K. Fukui, T. Enoki, Phys. Rev. B 73 (2006) 125415.

[16] S. Okada, A. Oshiyama, Phys. Rev. Lett. 87 (14) (2001) 146803.

[17] Y. Shimomura, Y. Takane, K. Wakabayashi, J. Phys. Soc. Jpn. 80 (2011) 054710 .

[18] A. Yamanaka and S. Okada, Carbon, 96 (2016) 351.

[19] J. Wu, W. Pisula, and K. Müllen, Chem. Rev. 107 (2007) 718 .

[20] K. Müllen and J. P. Rabe, Acc. Chem. Res. 41 (2008) 511.

[21] M. Fujihara, Y. Miyata, R. Kitaura, Y. Nishimura, C. Camacho, S. Irle, Y. Iizumi, T. Okazaki, and H. Shinohara, J. Phys. Chem. C 116 (2012) 15141.

[22] H.E. Lim, Y. Miyata, M. Fujihara, S. Okada, Z. Liu, Arifin, K. Sato, H. Omachi, R. Kitaura, S. Irle, K. Suenaga, and H. Shinohara, ACS Nano, 9 (2015) 5034.

[23] J. M. Robertson and J. G. White, J. Chem. Soc. 607 (1945).

[24] J. K. Fawcett and J. Trotter, Proc. Royal Soc. of London. Series A 289 (1966) 366.

[25] W. E. Barth and R. G. Lawton, J. Am. Chem. Soc. 88 (1966) 380.

[26] L. T. Scott, M. M. Hashemi, D. T. Meyer, and H. B. Warren, J. Am. Chem. Soc. 113 (1991) 7082.

[27] Y. Iizumi and T. Okazaki, submitted to Chem. Commun.

[28] K. Kusakabe, K. Wakabayashi, M. Igami, K. Nakada, and M. Fujita, Mol. Cryst. Liq. Cryst. 305 (1997) 445.

[29] S. Okada and T. Kawai, Jpn. J. Appl. Phys. 51 (2012) 02BN05. 
[30] P. Hohenberg and W. Kohn, Phys. Rev. 136 (1964) B864.

[31] W. Kohn and L. J. Sham, Phys. Rev. 140 (1965) A1133.

[32] Y. Morikawa, K. Iwata, and K. Terakura, Appl. Surf. Sci. 169-170 (2001) 11.

[33] J. P. Perdew, K. Burke, and M. Ernzerhof, Phys. Rev. Lett. 77 (1996) 3865.

[34] J. W. Jiang, J. S. Wang, and B. Li, Phys. Rev. B 80 (2009) 113405. 